\title{
Tumor characteristics and surgical outcome in incidentally discovered pheochromocytomas and paragangliomas
}

\author{
Xinlei Chen*, Liru Hu*, Caojie Liu, Guangcheng Ni and Yuwei Zhang \\ Department of Endocrinology and Metabolism, West China Hospital of Sichuan University, Chengdu, Sichuan, China \\ Correspondence should be addressed to Y Zhang: doczhangyuwei@sina.com \\ *(X Chen and L Hu contributed equally to this work)
}

\begin{abstract}
Objective: The proportion of incidentally discovered pheochromocytomas and paragangliomas (PPGL) has increased over time. However, our knowledge of them is quite limited. The purpose of this retrospective study is to generalize the commonalities in incidentally discovered PPGL, offer evidence for clinical diagnosis and management. Methods: Five hundred twenty-six patients were included in our study after filtration from the database of West China Hospital of Sichuan University between May, 2007 and December, 2016. Among the patients, 148 of them were incidental findings and 378 of them were suspected findings. All patients' demography and tumor characteristics were recorded in detail, especially hemodynamic records and hormonal assays. The reasons for taking radiography were also collected. Most patients received preoperative medical preparation. Intraoperative and postoperative courses as well as surgical outcomes were also analyzed to identify differences between incidental findings and suspected findings. Results: Incidentally discovered PPGL took up $28.1 \%$ of the study population. Suspected PPGLs had a higher prevalence of hypertension, lower proportion of non-functioning PPGL, higher prevalence of MEN2 and better post-surgical blood pressure recovery than incidental finding group. However, patients in the incidental finding group showed no significant difference in preoperative blood pressure and hormonal assays with suspected findings in metaphrine and normetaphrine in plasma and urine $(P>0.05)$. Conclusions: Due to the development of technology, more PPGLs are discovered incidentally. Considering the tumor characteristics and surgical outcome, surgical decisions should be made more cautiously.
\end{abstract}

\author{
Key Words \\ - pheochromocytoma \\ - paraganglioma \\ - adrenal incidentaloma \\ - preoperative care \\ - retrospective study
}

\section{Introduction}

Pheochromocytoma and paraganglioma (PPGL) are tumors composed of chromaffin cells, which commonly produce one or more catecholamines: epinephrine, norepinephrine and dopamine. These tumors are rarely silent, and they are categorized into two types based on their origin; pheochromocytomas (tumors from the adrenal medulla) and paragangliomas (tumors from the ganglia of the sympathetic chain in the thorax, abdomen and pelvis) (1). Paragangliomas can also arise from parasympathetic ganglia located along the glossopharyngeal and vagal nerves in the neck and at the base of the skull (2). The latter paragangliomas are usually non-secreting tumors (2).

Most PPGLs are hypersecreting tumors with high cardiovascular morbidity and mortality if left untreated; thus, diagnosis and treatment of the disease in an https://ec.bioscientifica.com

https://doi.org/10.1530/EC-18-0268 (c) 2018 The authors Published by Bioscientifica Ltd

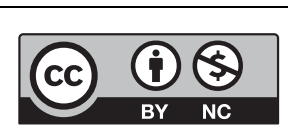

This work is licensed under a Creative Commons Attribution-NonCommercial 4.0 International License.
Endocrine Connections (2018) 7, 1142-1149 
early stage is of utmost importance. Although typical symptoms such as headaches, palpitations and sweating can provide clues for PPGL, owing to the unpredictable episodic secretion of catecholamines, these symptoms can present with varied manifestations, rendering accurate diagnosis difficult (3). Previous studies indicate a delay of 1-3 years and even miss in the diagnosis of PPGL (4). Furthermore, it is reported that more than 25\%, and up to $64 \%$ of pheochromocytomas are incidentally diagnosed $(5,6)$. These tumors are called 'incidentalomas.' Pheochromocytomas comprise $2-18.9 \%$ of all adrenal incidentalomas and are mainly diagnosed through CT taken for unrelated reasons (7).

While PPGLs are mostly benign, surgery is still usually recommended (8). Understanding of intraoperative hemodynamic instability (IHD), intraoperative and postoperative complications and mortality are vital to successfully evaluating perioperative outcomes. To decrease the risk of IHD as well as surgical complications and mortality, all patients are recommended to undergo a preoperative medical preparation, including patients with tumors rising from parasympathetic ganglia, since they can also release significant amounts of catecholamines during surgery (9).

With the increasing prevalence of incidentally diagnosed PPGL, incidentalomas have drawn more attention from clinical practitioners. The general idea of adrenal incidentalomas has been fully discussed in a large number of articles, whereas basic studies of incidentalomas in PPGL are limited. We found a limited number of articles describing the characteristics of pheochromocytoma as an incidentaloma, and these study populations were quite small $(6,10)$. Thus, the aim of this retrospective study is to generalize the commonalities of incidentaloma in PPGL from a large cohort with regard to their tumor characteristics, perioperative outcomes and prognoses, while providing evidence for clinical diagnosis and management.

\section{Methods and materials}

The study was approved by Sichuan University of West China Hospital GCP Center - a National Clinical Trials Agency.

Written consent has been achieved from all patients and subjects after full explanation of the purpose of the study.

From May 2007 to December 2016, we identified 743 patients diagnosed as PPGL from the database of West
China Hospital of Sichuan University. For the purpose of this study, only patients whose diagnoses were confirmed as PPGL and underwent surgery were included. Those whose records were incomplete or did not undergo surgery were excluded. After filtration, 526 patients were included in this retrospective study according to the inclusion and exclusion criteria.

All of the patients' diagnoses were confirmed by postoperational pathology. Detailed personal history, physical examination, preoperative and postoperative systolic and diastolic blood pressure and heart rate, biochemical analysis were reviewed. Biochemical analysis included metanephrine and normetanephrine in plasma and 24-h urine collection for 1 day. The upper limit of $\mathrm{MN}$ in plasma is $90 \mathrm{ng} / \mathrm{L}$, and the upper limit of NMN in plasma is $200 \mathrm{ng} / \mathrm{L}$. The normal range of total urine metanephrines is $42 \mu \mathrm{g} / 24 \mathrm{~h}$.

In our study, PPGLs were divided into two groups: 'Incidental findings' was used to define tumors detected unanticipated, usually through physical examination or image performed for an unrelated reason. In China, an ultrasonography of the abdomen is always included in the routine physical examination, and sometimes CT will be included. 'Suspected findings' was used to describe patients who present to the hospital with typical symptoms of PPGL. IHD includes hypertensive (intraoperative SBP $>160 \mathrm{mmHg}$ ) episodes and hypotensive (intraoperative mean artery pressure $<60 \mathrm{mmHg}$ ) episodes.

Most patients underwent preoperative medical management to reduce the risk of IHD (11). The dosage and application of anti-hypertensive drugs had been demonstrated in our previous study (12). In general, we used $\alpha$-blockade as suggested to normalize the blood pressure (13). For some patients with evidence of preoperative tachycardia or arrhythmia, $\beta$-blockade was applied. A high-salt diet was suggested by the guideline to prevent postoperative hypotension; however, salt was not much increased in their diet due to the adequate supplement from Chinese people's average salt intake, which had decreased over time but still held an intake of $9.0 \pm 2.0 \mathrm{~g} /$ day $(14)$.

Operations were carried out in all patients in our study when they achieved blood pressure normalization after preoperative management, which was defined by a target preinduction BP of 130/85 mmHg (15). Surgical approaches were determined by the location of the tumors, for patients whose tumors located in adrenal glands, open adrenalectomy was operated, for extra-adrenal tumors, laparotomy was carried out. Blood pressure and heart rate were monitored discreetly through the surgical duration https://ec.bioscientifica.com

https://doi.org/10.1530/EC-18-0268 (c) 2018 The authors Published by Bioscientifica Ltd
This work is licensed under a Creative Commons Attribution-NonCommercial 4.0 International License. 
by anesthesiologists, vasodilator was applied when systolic blood pressure was beyond $180 \mathrm{mmHg}$ during the operation.

After the operation, patients were sent to postanesthesia care unit (PACU) for better postoperative observation. Once the postoperative surgical complications occurred, patients would be sent to intensive care unit (ICU) for further arrangement. All notes from PACU and ICU were collected. Postoperative blood pressure, hormonal assays, recovering time and postoperative hospitalization duration were also achieved from daily progress notes. The time patients recovered was defined by relief of pain and without fatigue or sleeping disturbances (16).

The malignancy was determined by metastases in bone, liver and lymph nodes. And according to the 4th edition of World Health Organization classification, all PPGLs could have metastatic potential (2). Postoperative follow-up data were retrieved from 439 patients (128 from incidental findings and 311 from suspected findings, median follow-up 44 months, range 14-100 months) to observe the recurrence and metastases. The strategy for post-surgical screening contained annual abdominal CT and measurement of hormonal assays.

Frequency and percentage were calculated for descriptive statistics. Mean and standard deviation (STDEV) were calculated for continuous variables. For comparing nominal variables, difference was determined by chi-squared tests or Fisher exact test. As for continuous variables, Student-Newman-Keuls test or Mann-Whitney $U$ test were applied. Significance was accepted if $P<0.05$. All statistical analysis was performed using SPSS 22 (IBM Corporation).

\section{Results}

\section{Patient and tumor demography}

The patients were aged between 14 and 79 years, the mean age was $45.5 \pm 14.4$ years. 273 (51.9\%) of the patients were females. The delay of the diagnosis of suspected findings was counted by the time between initial symptoms and final diagnosis, reporting a length of 3.5 years in average and its standard deviation was 5.7 years, with a median of 1.0 years (range: $0.05-30$ years). All patients' diagnoses were confirmed through postoperative histological examination by experienced pathologists.

Average tumor diameter was $4.9 \pm 2.3 \mathrm{~cm}$ and 370 of the tumors $(70.3 \%)$ had a size over $4 \mathrm{~cm}$. Statistics of tumor diameter were collected from surgery records to assure the accuracy. Four hundred fifty patients' (85.7\%) tumors were unilateral, no significant difference in left or right. Forty-six $(8.7 \%)$ tumors were paragangliomas, which grew outside the adrenal gland, 14 of them were in pelvis, 22 in abdomen and 10 in thorax. None of them were found in head and neck.

Among all patients, 337 (64.1\%) were documented with hypertension, whereas 289 (35.9\%) of them were normotensive. Of the hypertensive patients, 233 patients (69.1\%) were persistent, 104 (30.9\%) of the patients were paroxysmal, experiencing unstable hypertensive episodes and large fluctuation in SBP from 110 to $240 \mathrm{mmHg}$. Of all patients, 335 (63.7\%) showed classic triad of PPGL, i.e., headaches, sweating and palpitation. Pheochromocytoma crisis was observed in 185 patients (35.2\%), which usually appeared as hypertensive crisis, with paroxysmal hypertension, pale complexion and palpitation and so forth. However, 195 (37.1\%) of them were non-functioning tumors and $64(12.2 \%)$ of them were non-functioning tumors with a diameter lower than $4 \mathrm{~cm}$.

\section{Incidental findings}

One hundred forty-eight patients (28.1\%) were incidentally discovered, through abdominal, thoracic or pelvic imaging taken routinely or for other reasons. The reasons for imaging were listed in Table 1 . The masses were detected initially through color Doppler ultrasonography in 59 patients $(11.2 \%)$, through computerized tomography in 78 patients $(14.8 \%)$ and 11 patients $(2.1 \%)$ took both examination and spotted abnormality in adrenal or extraadrenal area. All patients received CT scanning after the discovery of masses for further evaluation. Analyzing the composition ratio of patients each year, an increasing tendency of incidentally discovered PPGL can also be observed (Fig. 1).

Tumor characteristics, clinical manifestations and hormonal assays were analyzed in detail between incidental findings and suspected findings, the results were shown in Table 2. More of the incidental findings were non-functioning tumors $(58.8 \%$ in incidentaloma and $28.6 \%$ in suspected findings, $P<0.001$ ), without apparent increase in normetanephrine or metanephrine secretion, their hormonal levels were within normal range. Also, fewer incidental findings were documented with hypertension $(P<0.001)$. Interestingly, though mean value of hormonal assays of incidental findings was lower than suspected findings, significant statistical difference was not observed between groups $(P>0.05$ in 
Table 1 The initial reasons for patients with incidentally discovered PPGL underwent imageological examination.

\section{Reasons for imaging}

Imaging included in routine physical examination

Digestive disorder (gastrohelcosis, diarrhea etc.)

Acute inflammation (pleurisy, pancreatitis etc.)

Cardiomyopathy

Diabetes mellitus

Brain lesions

Liver disease (steatohepatitis, angioma etc.)

Valvular disease

Anxiety

Prostatic hyperplasia

Edema for unknown reason

Malignant tumor (nasopharyngeal carcinoma)

\begin{tabular}{c}
\hline N/percentage $(\%)$ \\
\hline $99 / 66.9$ \\
$9 / 6.1$ \\
$7 / 4.7$ \\
$7 / 4.7$ \\
$7 / 4.7$ \\
$7 / 4.7$ \\
$4 / 2.7$ \\
$2 / 1.4$ \\
$2 / 1.4$ \\
$2 / 1.4$ \\
$1 / 0.7$ \\
$1 / 0.7$
\end{tabular}

It should be highlighted that in China, patients sometimes undergo imaging like ultrasonography or CT during routine physical examinations, it doesn't suggest that they had found abnormalities in previous examinations.

all the four metanephrine secretion). Moreover, patients with suspected finding tumors were recorded with higher prevalence of multiple endocrine neoplasia type 2 (MEN2) $(P<0.001)$, a rare hereditary syndrome expressing a variety of mainly endocrine neoplasias including pheochromocytomas (17).

\section{Preoperative management}

Five hundred three patients $(95.6 \%)$ received preoperative medical treatment, in incidental finding group, 47 of them were treated with selective a blockade, 97 of them with non-selective a blockade, 2 of them were taking both and the rest 2 patients received no medication. In suspected finding group, 102 of them received selective alpha blockade, 246 of them were treated with nonselective alpha blockade, 9 with both medication and 21 without medical treatment. The length of preparation is $15.6 \pm 14.9$ days in incidental finding group and $13.5 \pm 12.3$ days in suspecting finding group. No statistical significance exists nor in medication choices $(P=0.127)$ or in the length of preparation $(P=0.281)$ between groups, which ruled out the influence of medication to the IHD and prognosis.

\section{Intraoperative and postoperative courses}

During the operation, blood pressure fluctuation was observed in 26 patients (4.9\%), 11 (11/148, 7.4\%) in incidental finding group and 15 (15/378, 4.0\%) in suspected finding group. There is no statistical difference between groups $(P=0.154)$.

No intraoperative or postoperative mortality was observed in the whole study population; however, six patients (1.1\%) suffered from postoperative bleeding and were transferred to surgical intensive care unit (SICU), four patients $(0.8 \%)$ presented with postoperative hypotension, four patients $(0.8 \%)$ lost consciousness after surgery and one patient $(0.2 \%)$ was anepia. All of them were within the suspected finding group. The patients were provided with in-time expectant treatment and their situation all became stable before discharge.

The mean postoperative recovering time was $3.8 \pm 2.4$ days in incidental finding group and $4.0 \pm 2.5$ days in suspected finding group. No significant difference existed between groups $(P=0.43)$. The postoperative hospitalization stay for incidental finding group and suspected finding group was $7.4 \pm 3.4$ days and $7.7 \pm 3.9$ days, respectively. No significance was found between groups $(P=0.59)$.

We focused on patients' change in blood pressure and hormonal assays to evaluate the surgical outcomes (Table 3). Twenty-six patients in the incidental finding group needed further management in blood pressure and the number was 68 in suspected finding group $(P=0.91)$.

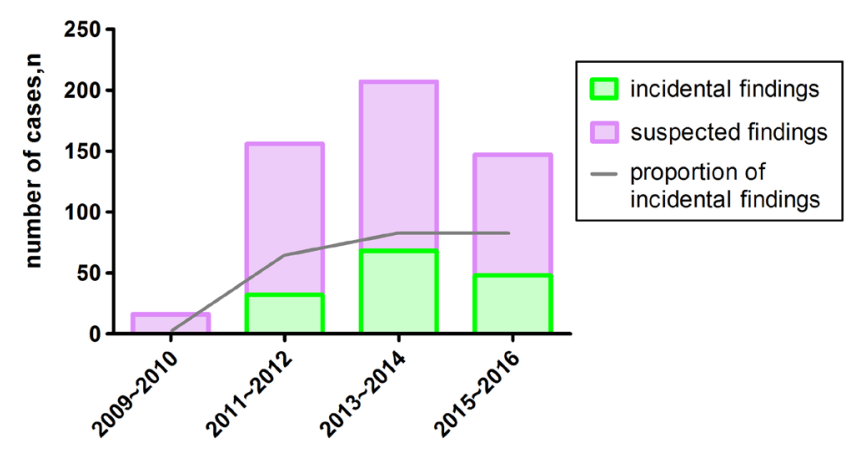

Figure 1

The tendency of incidentally discovered PPGL. The curve reveals an increasing tendency in the diagnosis of incidentally discovered PPGL over time. https://ec.bioscientifica.com

https://doi.org/10.1530/EC-18-0268 (c) 2018 The authors Published by Bioscientifica Ltd
This work is licensed under a Creative Commons Attribution-NonCommercial 4.0 International License. 
Table 2 Patient and tumor characteristics of incidental findings and suspected findings.

\begin{tabular}{l}
\hline Sex, male/female \\
Age (years) (average (medium)) \\
BMI $\left(\mathrm{kg} / \mathrm{m}^{2}\right)($ average (STDEV)) \\
Tumor side, unilateral/bilateral \\
Tumor diameter (average (STDEV)) \\
Documented with hypertension (\%) \\
Preoperative normetanephrine in plasma ( $\mathrm{ng} / \mathrm{L}$ ) (average \\
(STDEV)) \\
Preoperative metanephrine in plasma ( $\mathrm{ng} / \mathrm{L})$ (average \\
(STDEV)) \\
Preoperative normetanephrine in unrine $(\mu \mathrm{g} / 24 \mathrm{~h})$ \\
(average (STDEV)) \\
Preoperative metanephrine in urine $(\mu \mathrm{g} / 24 \mathrm{~h})$ (average \\
(STDEV)) \\
Non-functioning pheochromocytoma $(\%)$ \\
Multiple endocrine neoplasia II $(\%)$
\end{tabular}

It is noteworthy that postoperative SBP was significantly lower in suspected finding group, with $115 \pm 19.0 \mathrm{mmHg}$ in incidental finding group and $107 \pm 14.2 \mathrm{mmHg}$ in suspected finding group $(P<0.001)$, but both of them were within the normal range of SBP. Further, a correlation between preoperative SBP and postoperative SBP was analyzed and, obviously, suspected finding group shows a greater decline in SBP than incidental finding group (Fig. 2).

\section{Malignancy and follow-up}

A total of 31 patients $(6.0 \%)$ were classified as metastatic tumors, 16 patients (3.0\%) were diagnosed according to postoperative histological findings, 3 of them were incidentalomas and the rest of them were suspected findings. A long-term follow-up data was also achieved from the West China Hospital of Sichuan University's database and through phone calls. Fifteen patients who did not show signs of malignancy before discharge were diagnosed during follow-up by evidence of metastases in liver $(n=8)$, bone $(n=3)$ and lymph nodes $(n=4)$, five of them were in incidental finding group and ten of them were in suspected finding group. The overall prevalence of malignancy was $5.4 \%$ in incidental finding group and

\begin{tabular}{|c|c|c|}
\hline Incidental findings $(n=148)$ & Suspected findings $(n=378)$ & $P$ value \\
\hline $71 / 77$ & $182 / 196$ & 0.242 \\
\hline $47.8(49)$ & $44.4(45)$ & 0.246 \\
\hline $21.2(3.2)$ & $22.0(2.7)$ & 0.213 \\
\hline $138 / 10$ & $349 / 29$ & 0.721 \\
\hline $5.3(2.8)$ & $5.4(2.6)$ & 0.822 \\
\hline $48(32.4)$ & $289(76.5)$ & $<0.001$ \\
\hline $4363(11,454)$ & $5076(13,402)$ & 0.745 \\
\hline $280(362)$ & $427(981)$ & 0.319 \\
\hline $583(817)$ & 769 (1030) & 0.728 \\
\hline $87(216)$ & $141(218)$ & 0.548 \\
\hline $87(58.8)$ & $108(28.6)$ & $<0.001$ \\
\hline $2(1.5)$ & $33(8.7)$ & $<0.001$ \\
\hline
\end{tabular}

$4.4 \%$ in suspected finding group, no statistical significance was observed between groups $(P=0.77)$. Recurrence rate was $1.5 \%$ in incidental finding group and $2.6 \%$ in suspected finding group $(P=0.37)$.

\section{Discussion}

Our retrospective study revealed a high proportion of incidentally discovered PPGL, which makes up $28.13 \%$ of the entire study population. The frequency of incidentaloma has increased over time. This result agrees with the increasing tendency of incidentaloma in PPGL reported recently by other researchers (10). Routine physical examination accounts for nearly two-thirds of incidentalomas in PPGL, and better access to primary care may be among the reasons of increased incidental findings. Other explanations for increased diagnoses include advancements in diagnostic technology and the widespread application of radiography (i.e., CT, colorDoppler) and improved social awareness of healthcare. Further, 33.1\% of incidentalomas were discovered during treatment for unrelated diseases or symptoms, often in preoperative examination, which is fortuitous considering

Table 3 Postoperative hormonal assays of incidental findings and suspected findings.

\begin{tabular}{|c|c|c|c|}
\hline & Incidental findings $(n=148)$ & Suspected findings $(n=378)$ & $P$ value \\
\hline $\begin{array}{l}\text { Postoperative normetanephrine in plasma (ng/L) } \\
\text { (average (STDEV)) }\end{array}$ & 539 (339) & $898(1266)$ & 0.381 \\
\hline $\begin{array}{l}\text { Postoperative metanephrine in plasma (ng/L) (average } \\
(\text { STDEV)) }\end{array}$ & $147(145)$ & $103(122)$ & 0.329 \\
\hline $\begin{array}{l}\text { Postoperative normetanephrine in unrine }(\mu \mathrm{g} / 24 \mathrm{~h}) \\
\text { (average }(\mathrm{STDEV}))\end{array}$ & $44(46)$ & 95 (95) & 0.472 \\
\hline $\begin{array}{l}\text { Postoperative metanephrine in urine }(\mu \mathrm{g} / 24 \mathrm{~h}) \text { (average } \\
(\text { STDEV)) }\end{array}$ & $4.2(2.8)$ & $18.0(26.1)$ & 0.468 \\
\hline
\end{tabular}

https://ec.bioscientifica.com
https://doi.org/10.1530/EC-18-0268 $\quad \begin{gathered}\text { 2018 The authors } \\ \text { Published by Bioscientifica Ltd }\end{gathered}$




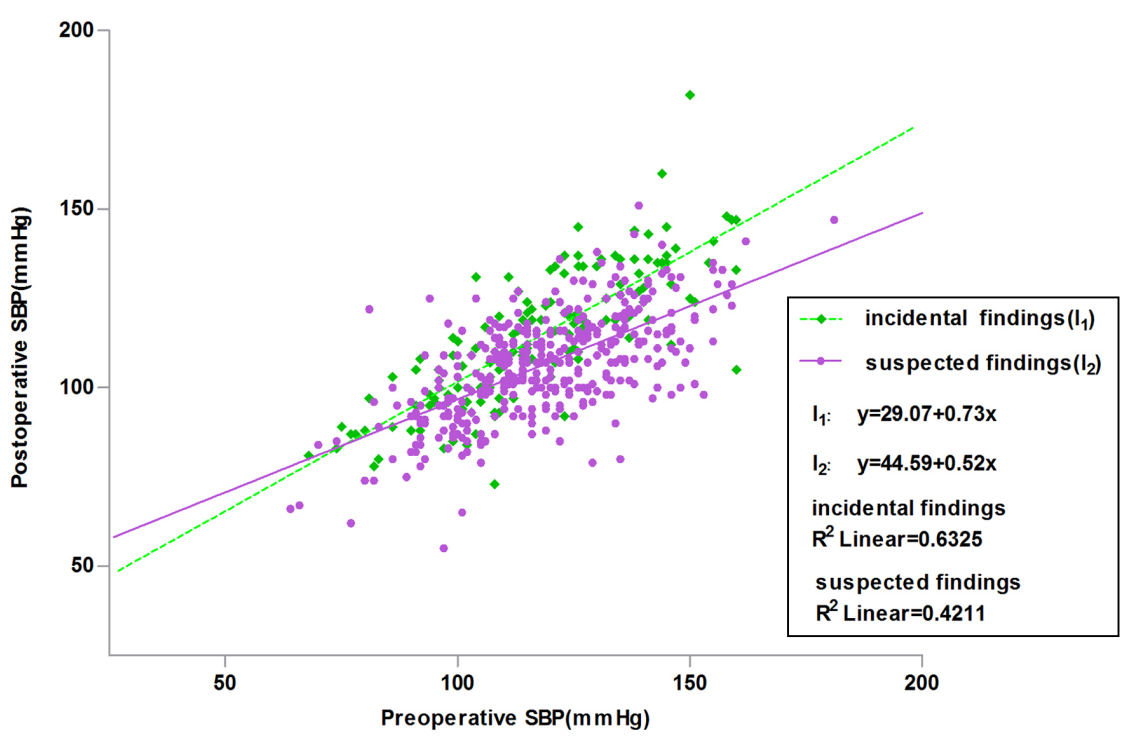

Figure 2

Bivariate scatter-plot showing preoperative SBP ( $X$-axis) vs postoperative SBP ( $Y$-axis). Patients in suspected findings group got a more obvious decline than those in incidental findings group. patients with undiscovered and unmanaged PPGL have a higher risk of perioperative morbidity and even worse, mortality $(18,19,20)$.

Our research also indicates a delay of $3.5 \pm 5.7$ years in the diagnosis of suspected PPGL, which is slightly longer than the interval indicated by earlier research (21). This could be a consequence of asymptomatic PPGL, since patients can present various symptoms such as tremors, nausea, anxiety or present asymptomatically. In our research, the classic triad presents in approximately two-thirds of patients, which is comparably high, but still suggests that typical symptoms are not sufficient to provide a gold standard for the diagnosis of PPGL. In our study, $64.1 \%$ of patients were documented with hypertension; $69.1 \%$ of them were persistent and $30.9 \%$ of them were paroxysmal. The proportion of normotensive patients was up to $23.5 \%$ in the suspected findings group, which is far higher than reported in the previous study, emphasizing that normotension does not rule out the presence of PPGL (21).

Kopetschke et al. and Amar et al. reported that patients with incidental findings were significantly older $(10,22)$. In contrast, patients with incidentalomas and suspected PPGL were similar in age in our study. Amar et al. also found that patients with incidentalomas had lower plasma catecholamine concentrations than patients with adrenergic symptoms (22). However, though incidentally discovered PPGLs showed a lower level of assayed hormones in our study, the result is not significant, which means incidentally discovered PPGLs did not differ from suspected tumors in catecholamine secretion when measured in plasma or in urine. Elevated catecholamines can lead to life-threatening symptoms; thus, physicians should not underestimate the perniciousness of incidentalomas.
In our research, we analyzed intraoperative and postoperative courses, as well as the surgical outcomes between incidental findings and suspected findings. Lanfont et al. reports that incidental findings may be less likely to develop perioperative hemodynamic instability (23). In our research, after similar preoperative management, no statistical difference was observed in the incidence of IHD. Instead, a lower risk of postoperative morbidity was observed in incidental findings. All the 15 patients suffering from postoperative morbidity had a tumor diameter of more than $4 \mathrm{~cm}$, which is in accordance with the theory that a larger tumor size indicates a higher risk of postoperative morbidity (18). However, since the statistical difference was not significant between the tumor diameters of the groups, we assumed the lower risk of postoperative morbidity in incidental findings might also be associated with the differences in hormone secretion of tumors, but this hypothesis requires further supporting evidence. Interestingly, the suspected findings group showed a greater decline in systolic blood pressure, which suggests a better outcome for suspected findings overall. To improve the outcome of incidental findings, a specific work-up for the management of incidentally discovered PPGL should be formulated.

A specific work-up for adrenal incidentalomas has been a research hotspot recently, where researchers have observed an excessive use of surgical intervention in adrenal incidentalomas and stress the importance of avoiding unnecessary surgery for benign lesions (24). Our study witnesses this phenomenon as well; while filtering our patients, a large number were ultimately diagnosed with non-secreting lesions such as steatadenoma, adrenal benign cysts. Further, among the patients included in our

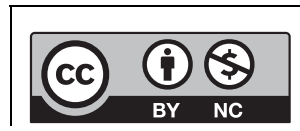

This work is licensed under a Creative Commons Attribution-NonCommercial 4.0 International License. 
study, which were all surgical patients, more than $10 \%$ of tumors had diameters smaller than $4 \mathrm{~cm}$, and were without significantly increased hormone levels in assays. Clinical practitioners should reflect on these findings and make more careful decisions in choosing surgery as a treatment option. Unless strongly demanded by patients, a conservative treatment and follow-up might be a better choice for small and non-secreting lesions. Some researchers argue for the standard that surgery should be restricted to patients with subclinical over-secreting lesions and lesions greater than $4 \mathrm{~cm}$ in diameter with atypical imaging patterns (25). Some studies hold the view that CT attenuation values and urine metaphrine together with urine normetaphrine as a combination is a convenient and effective indicator to distinguish pheochromocytoma from other adrenal tumors (26). It is always difficult to strike a balance between unnecessary damage caused by surgery and the risk of misdiagnosis, therefore clinical practitioners should fully use the means of diagnosis, and we are looking forward to new predictive parameters that might help physicians diagnose PPGL more precisely.

Notably, in our research, the suspected findings group contains more patients with MEN2, accompanied with other diseases such as medullary thyroid carcinoma (MTC) or hyperparathyroidism (HPTH). In accordance with other research, pheochromocytomas are usually diagnosed concomitantly or after the diagnosis of MTC, and it is the second most common presentation of MEN2 (27). MEN2 is known to associate with a mutation on the RET gene. It is also considered that the diagnosis of RET gene mutation status can contribute to the diagnosis of PPGL (28). Thus, earlier diagnosis and genetic testing should be advocated for and could be life-saving.

A long-term follow-up was also performed in our study. As the results show, no statistical difference exists in recurrence or metastases between groups, which indicating that incidentally discovered PPGL does not differ from suspected PPGL in their incidence of malignancy. Thus, it cannot be over-emphasized that a precautionary diagnosis should be made by physicians to avoid the misdiagnosis of PPGL. Furthermore, whether or not patients ultimately undergo surgery, a long-term follow-up should be provided to all PPGL patients.

There are, of course, several limitations to our study. First of all, as this is a retrospective study, preoperative medical management was determined by judgment of the attending physician, which might influence the intraoperative and postoperative courses. Fortunately, significant differences related to preoperative management were not observed in our analysis. Second, though we requested annual re-examinations, we lost several patients in the long-term follow-up due to changes in contact information along with poor patient compliance. The recurrence and metastatic rate might be higher if all possible data were collected. Moreover, genetic testing was not possible in our study due to the financial concerns of patients. Otherwise, patients might benefit more from receiving personalized treatments combining the status of their individual genotypes.

\section{Conclusion}

Owing to the development of imaging techniques and improved patient awareness, there is an increasing tendency toward incidentally discovered PPGL. Considering that no statistically significant differences were found in hormonal secretion and malignancy between incidentally discovered PPGL and suspected findings, physicians should be more alert for the diagnosis of PPGL. Further, a lower incidence of postoperative morbidity suggests that it is safer for patients to undergo surgery. Moreover, although the decline of SBP in incidental finding is not as great as that in the suspected finding group, both groups' SBP were within normal range, suggesting a promising outcome of operation in incidental findings. Still, as the understanding of incidentalomas in PPGL is limited, we hope a more specific work-up can be established to improve the diagnosis as well as the prognosis.

\section{Declaration of interest}

The authors declare that there is no conflict of interest that could be perceived as prejudicing the impartiality of the research reported.

\section{Funding}

This work was supported by Key Research Project of Sichuan Province Science and Technology Agency (grant numbers 18ZDYF1906).

\section{Acknowledgments}

Thanks to the dedication of patients and physicians as well as the support from West China Hospital of Sichuan Univerisity. This study was approved by Sichuan University of West China Hospital GCP Center.

\section{References}

1 Lenders JW, Duh QY, Eisenhofer G, Gimenez-Roqueplo AP, Grebe SK, Murad MH, Naruse M, Pacak K, Young WF \& Endocrine Society. Pheochromocytoma and paraganglioma: an endocrine society clinical practice guideline. Journal of Clinical Endocrinology and Metabolism 201499 1915-1942. (https://doi.org/10.1210/jc.2014-1498)

2 Lloyd RV, Osamura RY, Kloppel G \& Rosai J. WHO Classification of Tumours: Pathology and Genetics of Tumours of Endocrine Organs, 4th ed. Lyon, France: IARC Press, 2017.

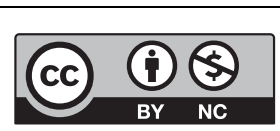

This work is licensed under a Creative Commons Attribution-NonCommercial 4.0 International License. 
3 Manger WM. The protean manifestations of pheochromocytoma. Hormone and Metabolic Research 200941 658-663. (https://doi. org/10.1055/s-0028-1128139)

4 Lenders JW \& Eisenhofer G. Pathophysiology and diagnosis of disorders of the adrenal medulla: focus on pheochromocytoma. Comprehensive Physiology 20144 691-713. (https://doi.org/10.1002/ cphy.c130034)

5 Noshiro T, Shimizu K, Watanabe T, Akama H, Shibukawa S, Miura W, Ito $\mathrm{S} \&$ Miura Y. Changes in clinical features and long-term prognosis in patients with pheochromocytoma. American Journal of Hypertension 200013 35-43. (https://doi.org/10.1016/S0895-7061(99)00139-9)

6 Henrik F, Magnus K \& Jan C. Initial clinical presentation and spectrum of pheochromocytoma: a study of 94 cases from a single center. Endocrine Connections 20187 186-192. (https://doi. org/10.1530/EC-17-0321)

7 Behbahani S, Mittal S, Patlas MN, Moshiri M, Menias CO \& Katz DS. 'Incidentalomas' on abdominal and pelvic CT in emergency radiology: literature review and current management recommendations. Abdominal Radiology 201742 1046-1061. (htps:// doi.org/10.1007/s00261-016-0914-5)

8 Liu H, Li B, Xuerong Y \& Huang Y. Preoperative risk factors for massive blood loss in adrenalectomy for pheochromocytoma. Oncotarget 20178 79964-79970. (https://doi.org/10.18632/ oncotarget.20396)

9 Weingarten TN, Welch TL, Moore TL, Walters GF, Whipple JL, Cavalcante A, Bancos I, Young WF, Gruber LM, Shah MZ, et al. Preoperative levels of catecholamines and metanephrines and intraoperative hemodynamics of patients undergoing pheochromocytoma and paraganglioma resection. Urology 2017100 131-138. (https://doi.org/10.1016/j.urology.2016.10.012)

10 Kopetschke R, Slisko M, Kilisli A, Tuschy U, Wallaschofski H, Fassnacht M, Ventz M, Beuschlein F, Reincke M, Reisch N, et al. Frequent incidental discovery of phaeochromocytoma: data from a German cohort of 201 phaeochromocytoma. European Journal of Endocrinology 2009161 355-361. (https://doi.org/10.1530/EJE-090384)

11 Williams DT, Dann S \& Wheeler MH. Phaeochromocytoma - views on current management. European Journal of Surgical Oncology 2003 29 483-490. (https://doi.org/10.1016/S0748-7983(03)00071-4)

12 Liu C, Lv Q, Chen X, Ni G, Hu L, Zhang Y \& Tong N. Preoperative selective vs nonselective $\alpha$-blockade in PPGL patients undergoing adrenalectomy. Endocrine Connections 20176 830-838. (https://doi. org/10.1530/EC-17-0232)

13 Lenders JW, Duh QY, Eisenhofer G, Gimenez-Roqueplo AP, Grebe SK, Murad MH, Naruse M, Pacak K, Young WF \& Endocrine Society. Pheochromocytoma and paraganglioma: an Endocrine Society Clinical Practice Guideline. Journal of Clinical Endocrinology and Metabolism 201499 1915-1942. (https://doi.org/10.1210/ jc.2014-1498)

14 Hipgrave DB, Chang S, Li X \& Wu Y. Salt and Sodium Intake in China. JAMA 2016315 703-705. (https://doi.org/10.1001/ jama.2015.15816)

15 Pacak K. Approach to the patient. Preoperative management of the pheochromocytoma patient. Journal of Clinical Endocrinology and Metabolism 200792 4069-4079. (https://doi.org/10.1210/jc.2007-1720)
16 Henrik K \& Jørgen BD. Anaesthesia, surgery, and challenges in postoperative recovery. Lancet 2003362 1921-1928. (https://doi. org/10.1016/S0140-6736(03)14966-5)

17 Serisha M, Frank W \& Lois M. The evolving clinical, genetic and therapeutic landscape of multiple endocrine neoplasia type 2 . Endocrine-Related Cancer 201825 E1-E4. (https://doi.org/10.1530/ ERC-17-0488)

18 Naranjo J, Dodd S \& Martin YN. Perioperative management of pheochromocytoma. Journal of Cardiothoracic and Vascular Anesthesia 201731 1427-1439. (https://doi.org/10.1053/j.jvca.2017.02.023)

19 Chang A, Silvay G \& Goldberg A. Intraoperative hemodynamic instability and diagnosis of pheochromocytoma during excision of adrenal incidentaloma with incomplete workup: a case report. Seminars in Cardiothoracic and Vascular Anesthesia 201722 328-331. (https://doi.org/10.1177/1089253217743338)

20 Hanna FWF, Issa BG, Sim J, Keevil B \& Fryer AA. Management of incidental adrenal tumours. BMJ 2018360 j5674. (https://doi. org/10.1136/bmj.j5674)

21 Lenders JW \& Eisenhofer G. Pathophysiology and diagnosis of disorders of the adrenal medulla: focus on pheochromocytoma. Comprehensive Physiology 20144 691-713. (https://doi.org/10.1002/ cphy.c130034)

22 Amar L, Servais A, Gimenez-Roqueplo AP, Zinzindohoue F, Chatellier G \& Plouin PF. Year of diagnosis, features at presentation, and risk of recurrence in patients with pheochromocytoma or secreting paraganglioma. Journal of Clinical Endocrinology and Metabolism 200590 2110-2116. (https://doi.org/10.1210/jc.20041398)

23 Lafont M, Fagour C, Haissaguerre M, Darancette G, Wagner T, Corcuff JB \& Tabarin A. Per-operative hemodynamic instability in normotensive patients with incidentally discovered pheochromocytomas. Journal of Clinical Endocrinology and Metabolism 2015100 417-421. (https://doi.org/10.1210/jc.2014-2998)

24 Haan RR, Visser JBR, Pons E, Feelders RA, Kaymak U, Hunink MGM \& Visser JJ. Patient-specific workup of adrenal incidentalomas. European Journal of Radiology 20174 108-114. (https://doi.org/10.1016/j. ejro.2017.08.002)

25 Gaujoux S, Aimé A, Assié G, Ciuni R, Bonnet S, Tenenbaum F, Bertherat J \& Dousset B. Adrenalectomy for incidentaloma: lessons learned from a single-centre series of 274 patients. ANZ Journal of Surgery 201788 468-473. (https://doi.org/10.1111/ans.14095)

26 Ohno Y, Sone M, Taura D, Yamasaki T, Kojima K, Honda-Kohmo K, Fukuda Y, Matsuo K, Fujii T, Yasoda A, et al. Evaluation of quantitative parameters for distinguishing pheochromocytoma from other adrenal tumors. Hypertension Research 201841 165-175. (https://doi.org/10.1038/s41440-017-0002-4)

27 Guerin C, Romanet P, Taieb D, Brue T, Lacroix A, Sebag F, Barlier A $\&$ Castinetti F. Looking beyond the thyroid: advances in the understanding of pheochromocytoma and hyperparathyroidism phenotypes in MEN2 and of non-MEN2 familial forms. EndocrineRelated Cancer 201825 T15-T28. (https://doi.org/10.1530/ERC-170266)

28 Joanna G \& Kym W. Patient quality of life and prognosis in multiple endocrine neoplasia type 2. Endocrine-Related Cancer 201825 T69-T77. (https://doi.org/10.1530/ERC-17-0335)

Received in final form 26 August 2018

Accepted 7 September 2018

Accepted Preprint published online 7 September 2018 https://ec.bioscientifica.com

https://doi.org/10.1530/EC-18-0268 (c) 2018 The authors

Published by Bioscientifica Ltd

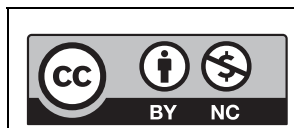

This work is licensed under a Creative Commons Attribution-NonCommercial 4.0 International License. 